\title{
A remote lab to support e-learning on FPAA
}

\author{
Manuel Felgueiras, André Fidalgo, Gustavo Alves \\ CIETI-LABORIS \\ Polytechnic Institute of Porto - School of Engineering \\ ISEP/IPP \\ Porto, Portugal \\ $\{$ mcf, anf, gca $\} @$ isep.ipp.pt
}

\author{
Guilherme Costa, Luis Schlichting, Golberi Ferreira \\ Instituto Federal de Santa Catarina \\ IFSC \\ Santa Catarina, Brazil \\ guilherme.costadasilva1996@gmail.com \\ \{schlicht, golberi\}@ifsc.edu.br
}

\begin{abstract}
Teaching analog and digital electronic subjects is not a trivial task because is necessary to use opposite methodologies. The electronic design in the digital field is centered in the use of microprocessor and FPGA based circuits using high level programing/configuring languages. The counterpart analog design is traditionally based in the use of elementary components associated with macroblocks such operational amplifiers in order to built-up the wanted mission circuit. Some few components, as the FPAA, are analogically configurable in a similar manner already used with the FPGA. However the use of this kind of components is not straightforward once is necessary to acquire some concepts not taught in the traditionally analog electronic classes.
\end{abstract}

The current work presents an innovative remote lab to support teaching of the FPAAs.

\section{Index Terms - Remote labs, FPAA, electronic design.}

\section{INTRODUCTION}

The function of the engineering profession is to manipulate materials, energy and information [1]. Teaching the several subjects includes information about theory that must be complemented by the experimentation in order to build the knowledge. This completion can be a demonstration for something learned or for verifying if some experiment meets the specifications. Laboratories are essential to demonstrate laws or concepts related to a given subject [2]. In the past, traditional laboratories classes were based in lab rooms oriented by a teacher. In the last years the Remote Labs rising in importance by several reasons and are now seen as one unavoidable alternative in practical teaching [3].

Electronic teaching includes the digital and analog but the Electronic Design strategies currently used in these two areas are completely opposite. In fact, the first is based in the use of programs/configuration using standard languages, in a hardware changeless approach. On the contrary, the second one is traditionally based on the use of typical circuit topologies adapted to each situation where each change is made by hardware modification.

Some components such the Field Programmable Analog Arrays (FPAA) [4,5], or the Programmable System-on-Chip (PSoC) [6] allow making the analog electronic design in a manner similar to the currently used in digital circuits, through a program/configuration. Differentially than the digital counterpart, the use of this components is not straight once involves the knowledge of some particular concepts.

This work presents a remote lab to support the eLearning of FPAA.

\section{ELECTRONIC TEACHING}

Teaching is structured activity that encompasses several issues such as the reuse earlier concepts to transmit new ones. One major limitation is that the several subjects to teach remain in a significant different state of development of its components and therefore impose different approach methodologies. This is the case of teaching electronics subjects where the digital electronic present a very high development state when compared by the analog electronic.

The first is based in the use of flexible circuits based in microprocessor (e.g. microcontrollers) or Field Programmable Gate Array (FPGA). The definition of the Mission Circuit is made by using normalized programing languages (e.g. C language) for microprocessors based circuits or Hardware Description Languages (HDL) (e.g. Verilog, VHDL) for FPGA. This methodology corresponds to the use of high level commands instructions which are associated to produce a program. This methodology is very appealing to the new generation of students and completely changing-less at hardware level. In this way, the user can take advantage from the regular improvements in components features such new powerful instructions, speed, size etc.

The second is traditionally based in the use of elementary components (e.g. resistors, capacitors, diodes, transistors, etc.) or macroblocks (e.g. Operational Amplifiers) that are associated to procedure the Mission Circuit. This methodology starts continually from the elementary and corresponds to constant reinvention is the wheel. As seen, the digital arena uses a top-down electronic design methodology whereas in the analog arena uses a bottom-up one. Being opposite, this often bring some confusing to the student that prefer to remain only in the digital arena, once is more high level and thus more close to the human way to think.

The FPAA is a configurable analog component that allows to be configured using the very same methodology used to the digital flexible circuits. The figure1 presents the FPAA development kit.

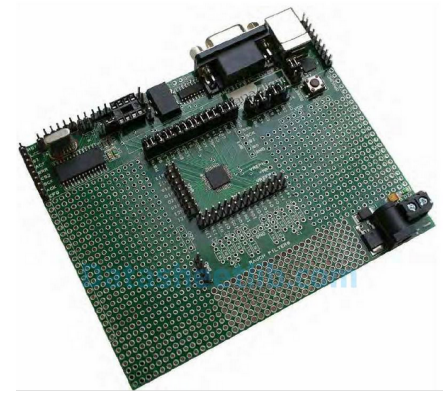

Figure 1. The FPAA development kit. 
However, the use of this components is not straightforward once present some drawbacks that should be taken in account to be possible the transition between the traditional manner of teaching analog design to the one using FPAA. It is necessary to establish some steps to make the use of the FPAA acceptable by the traditional student that comprehends the development of:

- A set of means to clarify concepts

- A remote lab to practice with FPAA

- A set of means to inform the student about his knowledge level

The first includes the development of writing notes, slides, flash movies, etc., to explain some notions related to analog design such ground (e.g. differences between ground and earth, power supply ground, analog ground, digital ground etc.), types of signals (digital, analog, quasi-digital), signals references (single-ended signal, double-ended signal), and switched-capacitor technology. All these concepts are important because they are intrinsically involved with the FPAA. For instance, it works with single supply and the analog ground is referred to a different voltage from the main power supply (e.g. analog ground $=1.5 \mathrm{~V}$ ). This part of the work is practically concluded and will be soon available to the community.

The second one is important to practice with FPAA without having the need to buy a development kit. This point will be clarified in a subsequence section.

The third one is related to the means that will be developed to help the student to verify if his skill level matches the intended and will be based in the use of the MOODLE.

\section{THE FPAA REMOTE LAB}

The developed FPAA remote lab intends to provide a simple platform to remotely configuring the FPAA and verify if the actual Mission Circuit matches the intended one. This platform includes several blocks as depicted in figure 2 .

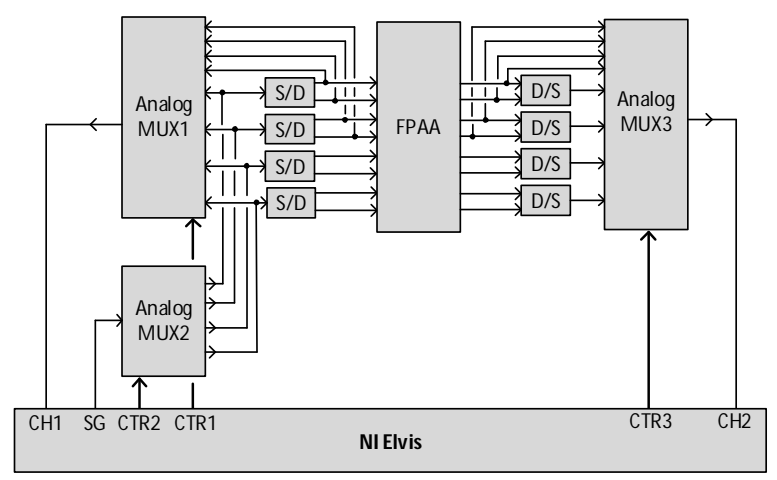

Figure 2. The FPAA remote lab infrastructure.
The core of all circuit is the FPAA. Its input lines are preceded by the circuit S/D that converts single-ended signals into dual-ended signals. In the output we have the $\mathrm{D} / \mathrm{S}$ circuits that have the reverse function of the $\mathrm{S} / \mathrm{D}$, i.e., converts dual-ended signals into single-ended signals. The NI Elvis station is used to supply test signals from its internal Signal Generator (SD), to observe the input and output signal to/from the FPAA and also to control the several analog multiplexers. The MUX2 select which FPAA input will be connected to the SG. The MUX1 and MUX3 selects the FPAA input and output that will be connected to the channel one (CH1) and channel two (CH2) of the internal NI ELVIS oscilloscope.

The presented platform allows developing a significant number of experimental tests in the FPAA.

\section{CONCLUSION}

Teaching analog electronic design subjects involves a methodology other than the used in the digital electronic design mainly caused by the different state of development of the electronic components from each arena. In the digital is currently used the top-down approach whereas the bottom-up into the analog. The first is easier to use not only because is more close to the human brain but also is hardware changing-less. This work presents a remote lab to support learning in FPAA. After having attained some basics of FPAA, the user will be able to verify if the developed configuration matches the intended one.

\section{REFERENCES}

[1] Feisel, L., Rosa, A., "The role of the Laboratory in the Undergraduate Engineering Education", Journal of Engineering Education, January, 2005.

[2] Silva, R. ; Moreira, R. ; Gouveia, L. ; Felgueiras, M., "Unblinding a Braille machine - A case study of reverse problem-based learning". Proceedings of the 2013 1st International Conference of the Portuguese Society for Engineering Education (CISPEE). October 31th-November 01st, Porto, 2013.

[3] Alves, G.R., Marques, M.A., Viegas, C., Costa Lobo, M.C. "Using VISIR in a large undergraduate course: Preliminary assessment results", Proceedings of Global Engineering Education Conference (EDUCON), 2011.

[4] Becker, J, Trendelenburg, S., Henrici,F., Manoli, Y. , "A field programmable Gm-C filter array (FPAA), Proceedings of the Second NASA/ESA Conference on Adaptive Hardware and Systems(AHS), 2007.

[5] Anadigm, http://www.anadigm.com/, Visited in January 2015.

[6] Cypress - Programmable System-on-Chip, http://www.cypress.com/psoc/? source=CY-ENG-HEADER, Visited in January 2015. 